\title{
X-RAY PHOTOELECTRON STUDY OF Yb-DOPED InP
}

\author{
R.J. IWanowski, J.W. SobczaK* and Z. KaLIŃSKI \\ Institute of Physics, Polish Academy of Sciences \\ Al.Lotników 32/46, 02-668 Warsaw, Poland \\ *Institute of Physical Chemistry, Polish Academy of Sciences \\ Kasprzaka 44/52, 01-224 Warsaw, Poland
}

\begin{abstract}
X-ray photoelectron spectra of core levels are reported for InP:Yb. Crystalline InP, doped with $\mathrm{Yb}$ to a level of 0.5 at.\%, was grown by the synthesized solute diffusion method. An analysis of the core-level spectra of the constituent components, i.e. In $3 d_{5 / 2}$ and $\mathrm{P} 2 p$, revealed a minor influence of the surface oxide species, mainly in the phosphate-like form. The spectrum of the $\mathrm{Yb} 4 d$ core level was also recorded. The energy of the $\mathrm{Yb} 4 d_{3 / 2}$ peak was found identical to that in $\mathrm{Yb}$ metal, whereas the $4 d_{5 / 2}$ peak was found to be shifted to higher binding energies. This effect was found comparable to the case of advanced oxidation of $\mathrm{Yb}$ thus confirming its high reactivity, even as a bulk dopant. The data give also a rare experimental example of detection of bulk dopant atoms in a semiconductor matrix by X-ray photoelectron spectroscopy at the limit of detectability.
\end{abstract}

PACS numbers: 71.55.Eq, 79.60.Bm

\section{Introduction}

In the decade of the eighties, indium phosphide has proved to be a very promising semiconductor material for various applications owing to its intrinsic properties, such as high electron mobility and high radiation tolerance. This obviously implied the interest in its potential applications, such as high-speed electronic devices, high-performance space solar cells and integrated optoelectronic devices. In the latter case, the interest has been additionally enhanced after successful incorporation of $\mathrm{Yb}$ into InP by bulk [1] or epitaxial growth [2], which led to the observation of the $\mathrm{Yb}^{+3}$-related electroluminescence in the emission band around $1.24 \mathrm{eV}[3,2]$.

The perspective of electronic device applications has been also stimulating for studies of InP surface preparation with the use of X-ray photoelectron spectroscopy (XPS), e.g. [4-6]. In the case of Yb-doped InP, there is a lack of reports concerning XPS investigations (see Ref. [7]). Hence, the goal of the present paper was to provide preliminary results of an XPS characterization of InP:Yb. 


\section{Experiment}

Bulk InP crystals were grown by the synthesized solute diffusion (SSD) method [8] at the Institute of Physics, Polish Academy of Sciences. 0.5 at.\% of $\mathrm{Yb}$ metal was added to the starting material to grow an Yb-doped crystal. The growth temperature was $850^{\circ} \mathrm{C}$. The obtained crystalline ingot was $4 \mathrm{~cm}$ long with a diameter of $1 \mathrm{~cm}$. The sample $(1 \mathrm{~mm}$ thick) prepared for this experiment was cut from the middle part of the ingot, perpendicularly to the main axis. Its surface was subjected to fine polishing and was finally etched in a $1 \% \mathrm{Br}_{2}$-methanol solution. Electron microprobe (JEOL JSM-50A) analysis enabled us to estimate that the real $\mathrm{Yb}$ concentration in the sample remains below its assumed value ( 0.5 at.\%).

Core-level X-ray photoelectron spectra were measured at room temperature in a vacuum generator (VG) Escalab 210 spectrometer using an $\mathrm{Al} K_{\alpha}(1486.6 \mathrm{eV})$ irradiation source. The photoelectrons were detected normal to the surface.

\section{Results and concluding remarks}

Core-level XPS spectra of the constituent elements of InP, namely P $2 p$ and In $3 d_{5 / 2}$, are shown in Figs. 1 and 2a, respectively.

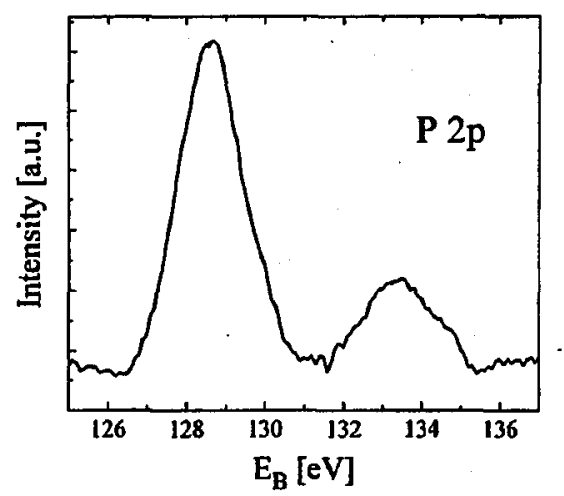

Fig. 1. Photoelectron spectrum of the $\mathrm{P} 2 p$ level excited with $\mathrm{Al} K_{\alpha}$ radiation from the InP surface.

In particular, the $\mathrm{P} 2 p$ spectrum (Fig. 1) is readily observed as a doublet. One can distinguish there the main peak at $128.7 \mathrm{eV}$ ascribed to $\mathrm{P}$ in InP (bulk component), and the minor peak around $133.6 \mathrm{eV}$ (surface component), which is indicative of phosphorus bound in phosphates, introduced by surface oxidation (see $[4,5,7])$.

The photoelectron spectrum of the In $3 d_{5 / 2}$ core level (Fig. 2a) exhibits an asymmetric shape, characteristic for an overlap of bulk- and surface-component peaks $[4,5]$. Its numerical analysis, performed by fitting with Gaussian-Lorentzian peaks, enabled us to distinguish the dominant bulk-related peak $\left(E_{\mathrm{B}}=444.2 \mathrm{eV}\right)$ from the weaker surface-related contribution $\left(E_{\mathrm{B}}=445.1 \mathrm{eV}\right)$.

A similar analysis was also performed for the $O 1 s$ photoelectron spectrum, where four constituent subpeaks were found (Fig. 2b). 

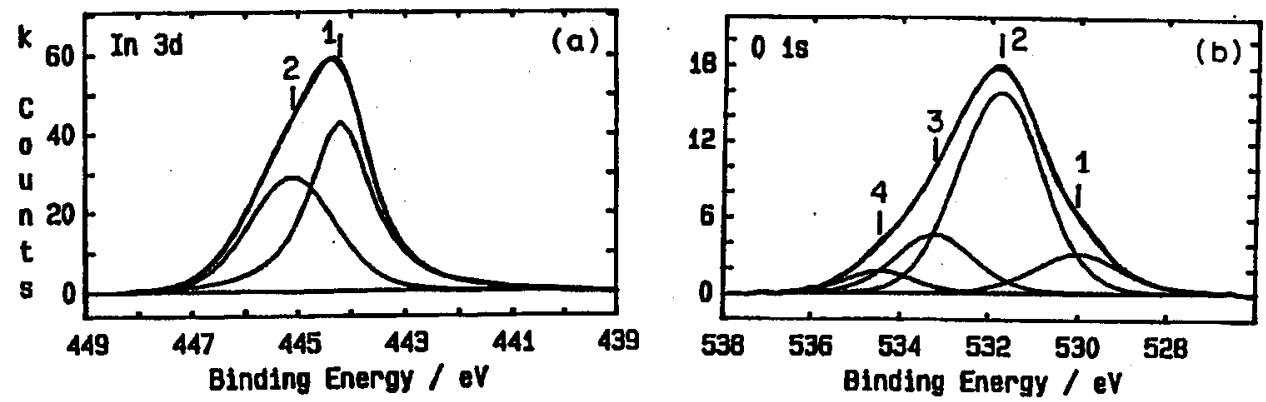

Fig. 2. Photoelectron spectra of In $3 d_{5 / 2}$ (a) and $\mathrm{O} 1 s$ core (b) levels in InP and results of their numerical analysis (for details see text and Table).

TABLE

Results of a numerical analysis of the In $3 d_{5 / 2}$ and $\mathrm{O} 1 \mathrm{~s}$ core-level photoelectron spectra measured from the InP:Yb surface. FWHM denotes the full line width at half-maximum.

\begin{tabular}{l|c|c|c|c}
\hline \hline Core level & Peak No. & $E_{\mathrm{B}}[\mathrm{eV}]$ & FWHM [eV] & Area [\%] \\
\hline In $3 d_{5 / 2}$ & 1 & 444.2 & 1.38 & 55 \\
& 2 & 445.1 & 1.96 & 45 \\
\hline O $1 s$ & 1 & 530.0 & 2.35 & 13 \\
& 2 & 531.8 & 2.29 & 64 \\
& 3 & 533.2 & 2.16 & 17 \\
& 4 & 534.5 & 1.83 & 6
\end{tabular}

Table summarizes the results of the numerical analysis of both the In $3 d_{5 / 2}$ and $\mathrm{O} 1 s$ XPS peaks, retaining the same notation of subpeaks as in the respective figures.

Taking into account the above evidence (Figs. 1 and 2, Table) and the literature data [5] one can conclude that in a surface- and subsurface region of $\mathrm{InP}$, accessible to XPS, there is a noticeable (although minor) influence of different native oxide species.

In particular, the peak $E_{1}\left(\mathrm{O}_{1} s\right)$ is due to the presence of indium oxide $\left(\mathrm{In}_{2} \mathrm{O}_{3}\right)$ [5]. The parameters (energy, FWHM) of the peak 2 from the $\mathrm{O} 1 s$ spectrum indicate that it probably results from superposition of the contributions given by $\operatorname{In}(\mathrm{OH})_{3}, \operatorname{In}(\mathrm{OH})_{3} \cdot x \mathrm{H}_{2} \mathrm{O}, \mathrm{In}_{2} \mathrm{O}_{3}$ as well as the phosphate species like $\mathrm{InPO}_{4}$ and $\mathrm{InPO}_{4} \cdot x \mathrm{H}_{2} \mathrm{O}$ [5]. The $E_{3}(\mathrm{O} 1 s)$ peak (Table) can be attributed to the existence of $\operatorname{In}\left(\mathrm{PO}_{3}\right)_{3}$, that also contributes to the $E_{2}(\mathrm{O} 1 s)$ peak [5]. The weak $E_{4}(\mathrm{O} 1 s)$ peak also points at the residual admixture of $\operatorname{In}\left(\mathrm{PO}_{3}\right)_{4}[5]$.

The peak 1 of the In $3 d_{5 / 2}$ spectrum (Table) has relatively low FWHM $(1.38 \mathrm{eV})$, that is typical for unperturbed bulk-related component [5]. On the other hand, broadening of the subpeak $E_{2}\left(\operatorname{In} 3 d_{5 / 2}\right)(\mathrm{FWHM}=1.96 \mathrm{eV}-$ Table) is indicative of the overlapping contributions from different (above-mentioned) 
oxides and phosphate-like species (see [5]). The split of $E_{2}$ from the $E_{1}$ peak is due to relatively higher (compared to phosphorus) electronegativity of oxygen [9], bound to In in the oxide or phosphate configuration.

The apparent existence of native oxides in the studied case is not surprising, since we intentionally avoided a surface-deoxidation etch in order to check the influence of partial surface oxidation on the binding of $\mathrm{Yb}$ in the InP lattice.

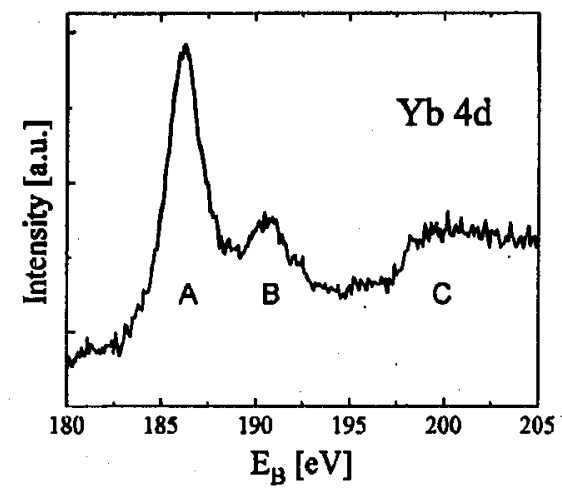

Fig. 3. Photoelectron spectrum of $\mathrm{Yb} 4 d$ levels taken from the InP:Yb surface.

The XPS spectrum of the $\mathrm{Yb} 4 d$ core-level is presented in Fig. 3. One can distinguish there the most pronounced peak (denoted by A) centered at $186.2 \mathrm{eV}$ and a weaker one (B) at $190.6 \mathrm{eV}$. There is also an additional broad feature (C) located around $199 \mathrm{eV}$. A comparison with earlier data $[10,7]$ enabled us to assign the peaks $\mathrm{A}$ and $\mathrm{B}$ with the spin-orbit split $4 d$ levels $\left(4 d_{5 / 2}\right.$ and $4 d_{3 / 2}$, respectively). The energy of the $4 d_{5 / 2}$ peak, $E_{\mathrm{B}}(\mathrm{A})$, (together with its intensity ratio vs. $4 d_{3 / 2}$ peak) is a specially useful indicator in monitoring the oxidation state $[7,10]$ and, in general, the valency state. The value of $E_{\mathrm{B}}(\mathrm{A})$ is found here to be shifted (from that of $\mathrm{Yb}$ metal) to higher binding energy, which coincides well with that of $\mathrm{Yb}_{2} \mathrm{O}_{3}[7]$. On the other hand, the energy of the peak $\mathrm{B}(190.6 \mathrm{eV})$ agrees very well with that of the corresponding $4 d_{3 / 2}$ peak of $\mathrm{Yb}$ metal $[10,7]$ and a partially oxidized $\mathrm{Yb}[10]$, whereas it slightly differs from that of pure $\mathrm{Yb}_{2} \mathrm{O}_{3}$ $\left(E_{\mathrm{B}} \cong 192 \mathrm{eV}[10,11]\right)$. Despite of its complexity, the spectrum considered (Fig. 3) seems indicative of the advanced oxidation stage of $\mathrm{Yb}$, with dominant +3 valency state, characteristic of $\mathrm{Yb}_{2} \mathrm{O}_{3}[7,10]$. This can be also supported by an appearance of the feature $\mathrm{C}$, which is usually attributed to the $4 f^{13}$ configuration of $\mathrm{Yb}$ ion (see also [12]). This enables us to judge that in our case of partially oxidized surfaceand subsurface region of $\mathrm{InP}$ crystal the $\mathrm{Yb}$ dopant ions are mainly bound to oxygen and form $\mathrm{Yb}_{2} \mathrm{O}_{3}$ clusters in the host matrix, thus confirming a very high reactivity of $\mathrm{Yb}$. The earlier EPR studies [13] performed for bulk InP:Yb crystals, grown by the same method, have proved that in the absence of oxygen an $\mathrm{Yb}^{3+}$ ion (electrically neutral with respect to $\mathrm{In}^{3+}$ ) occupies an In site in the lattice. Therefore, taking into account the oxygen concentration gradient in a subsurface region, one can not exclude here a certain influence of these latter $\mathrm{Yb}$ ions on 
the Yb $4 d$ XPS spectrum (Fig. 3), especially that the photoelectron signal was recorded in the maximum bulk sensitivity mode. This could be obviously verified when repeating the measurement for the clean surface of the same crystal.

Our data represent the first, although preliminary, XPS analysis of Yb impurities in InP. They are also a rare experimental example of the XPS detection of bulk dopant atoms in a semiconductor matrix in the doping range of $\lesssim 0.5$ at.\%, i.e. at the XPS detectability limit [14].

\section{Acknowledgement}

One of the authors (R.J.I) feels deeply indebted to Professor A. Jabłoński for enabling him to perform this experiment in his department.

\section{References}

[1] V.A. Kasatkin, V.F. Masterov, V.V. Romanov, B.E. Samorukov, K.F. Shtel'makh, Sov. Phys. Semicond. 16, 106 (1982).

[2] W.H. Haydl, H.D. Muller, H. Ennen, W. Korber, K.W. Benz, Appl. Phys. Lett. 46, 870 (1985).

[3] A.G. Dmitriev, L.F. Zakharenkov, V.A. Kasatkin, V.F. Masterov, B.E. Samorukov, Sov. Phys. Semicond. 17, 1201 (1983).

[4] J.H. Thomas, G. Kaganowicz, J.W. Robinson, J. Electrochem. Soc. 135, 1201 (1988).

[5] M. Faur, M. Faur, D.T. Jayne, M. Goradia, C. Goradia, Surf. Interface Anal. 15, 641 (1990).

[6] M. Faur, M. Faur, P. Jenkins, M. Goradia, S. Bailey, D. Jayne, I. Weinberg, C. Goradia, Surf. Interface Anal. 15, 745 (1990).

[7] J.F. Moulder, W.F. Stickle, P.E. Sobol, K.D. Bomben, Handbook of X-Ray Photoelectron Spectroscopy, Perkin-Elmer Corporation, Physical Electronic Division, Eden Prairie (Minnesota) USA 1994.

[8] K. Gillesen, A.J. Marshal, J. Hesse, in: Crystal Growth, Properties and Applications, Ed. H.C. Freyhardt, Springer, Berlin 1980, p. 49.

[9] L.N. Pauling, The Nature of the Chemical Bond, Cornell Univ., Ithaca (NY) 1960.

[10] S.B.M. Hagstrom, P.O. Heden, H. Lofgren, Solid State Commun. 8, 1245 (1970).

[11] A.J. Signorelli, R.G. Hayes, Phys. Rev. B 8, 81 (1973).

[12] J.S. Chung, E.J. Cho, S.-J. Oh, Phys. Rev. B 41, 5524 (1990).

[13] A. Stapor, J. Raczyńska, H. Przybylińska, A. Sienkiewicz, K. Fronc, J.M. Langer, Mater. Sci. Forum. 10-12, 633 (1986).

[14] T.J. Shaffner, Surf. Interface Anal. 14, 598 (1989). 